\title{
Complex ventricular tachycardia coexistent with myocardial bridging
}

\author{
(1) Sinan Cersit, ${ }^{1}$ (D) Mustafa Ozan Gursoy, ${ }^{2}$ (i) Lutfi Ocal ${ }^{1}$ \\ ${ }^{1}$ Department of Cardiology, Kosuyolu Kartal Heart Training and Research Hospital, Istanbul, Turkey \\ ${ }^{2}$ Department of Cardiology, Katip Celebi University, Ataturk Training and Research Hospital, Izmir, Turkey
}

\begin{abstract}
Sustained monomorphic ventricular tachycardia is rarely a concomitant condition with myocardial bridging for which no evidence-based medical management has yet been certainly described. Here, in this case study, we present a case of malignant ventricular arrhythmia that may be associated with the myocardial bridge on the coronary artery. The clinical management and medical treatment of the patient are discussed.
\end{abstract}

Keywords: Coronary angiography; myocardial bridging; sustained monomorphic ventricular tachycardia.

Cite this article as: Cersit S, Gursoy MO, Ocal L. Complex ventricular tachycardia coexistent with myocardial bridging. North Clin Istanb 2020;7(6):621-623.

$M$ yocardial bridging $(\mathrm{MB})$ is an inborn disorder defined by systolic compression of coronary artery lumen that normalizes during diastole. It may cause several manifestations, such as angina, arrhythmia and sudden death [1]. In this case study, we present a case of complex ventricular tachycardia (VT) in a patient with coronary $\mathrm{MB}$.

\section{CASE REPORT}

A forty-eight years-old man presented to the emergency department due to sudden onset of palpitations and dizziness. He had no coronary risk factors and prior history of cardiac disease. Heart rate was 155 beats/ minute, and blood pressure was 118/63 mm Hg. His physical examination was unremarkable. The electrocardiogram (ECG) demonstrated a wide-QRScomplex tachycardia compatible with a sustained monomorphic ventricular tachycardia (VT), which terminated spontaneously (Fig. 1). The next ECG revealed normal sinus rhythm. Laboratory analysis, including troponin, was normal, and there were no segmental wall-motion abnormalities in the advanced echocardiographic examination. Coronary angiography showed remarkable coronary compression in the middle segment of the left anterior descending artery (mid-LAD) during the systolic cardiac phase without significant coronary artery disease, indicating myocardial bridging (MB) (Fig. 2 and Video 1). A relationship was suspected between VT and MB after excluding other possible causes of VT. Cardiac magnetic resonance imaging (MRI) did not demonstrate zones of scar that could form the substrate for the sustained monomorphic VT. There were no further complaints and tachycardia with the initiation of medical therapy with $100 \mathrm{mg}$ metoprolol succinate. One month after this event, the patient was able to perform 10.1 METS on a Bruce protocol with a blunted maximal heart rate of 115 beats/minute on metoprolol and no perfusion defect was detected by single-photon emission com-

Received: January 11, 2019 Accepted: March 01, 2019 Online: March 26, 2019

Correspondence: Sinan CERSIT, MD. Kosuyolu Kartal Kalp Egitim ve Arastirma Hastanesi, Kardiyoloji Klinigi, Istanbul, Turkey. Phone: +90 2165150000 e-mail: sinancersit@hotmail.com

(c) Copyright 2020 by Istanbul Provincial Directorate of Health - Available online at www.northclinist.com 


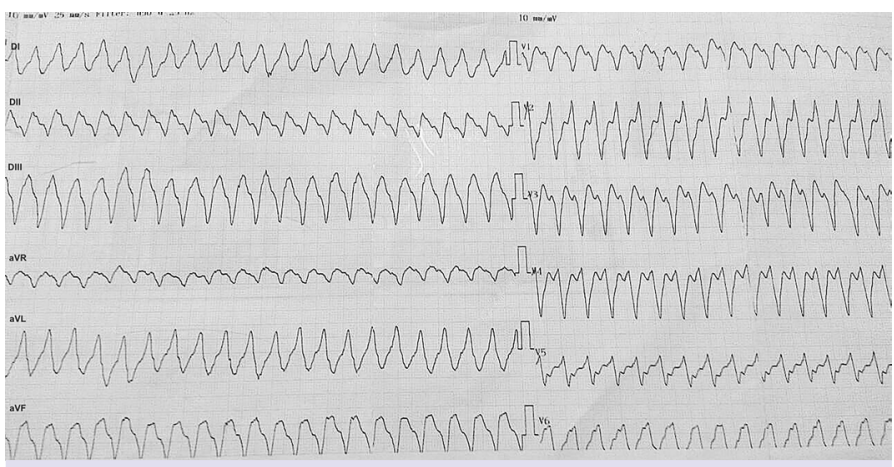

FIGURE 1. Wide-QRS-complex tachycardia compatible with sustained monomorphic ventricular tachycardia.

puted tomography. The patient's consent was obtained for this case report.

\section{DISCUSSION}

$\mathrm{MB}$ is an inborn abnormality that a certain segment of the epicardial coronary artery goes into the heart muscle band. $\mathrm{MB}$ is reported angiographically in $1.22 \%$ to $15 \%$ of patients and is usually localized to mid-LAD $[1,2]$. Although $\mathrm{MB}$ has been generally considered a benign disease, it may cause severe conditions, such as ischemia, acute coronary syndrome, arrhythmia (including supraventricular tachycardia and VT) and sudden cardiac death [3].

Delay in the duration of ventricular relaxation due to the prolongation of the $\mathrm{MB}$ contraction, especially with tachycardia episodes, may impair diastolic coronary perfusion. This pathologic condition may contribute to VT associated with $\mathrm{MB}[4]$.

Sustained monomorphic VT is a potentially lifethreatening arrhythmia that should be urgently evaluated for ischemic heart disease under the guidance of multimodality imaging, including advanced echocardiography, conventional coronary angiography and cardiac MRI [5].

Medical management with negative inotropic and chronotropic agents is considered as first-line therapy. Beta-blockers decrease in contractility and compression of the coronary arteries by lowering the heart rate and increasing diastolic interval. Thus, these agents are generally beneficial although they have not been studied in randomized controlled trials [6].

\section{Conclusion}

$\mathrm{MB}$ may be a cause of VT although it is generally accepted as a benign anomaly.
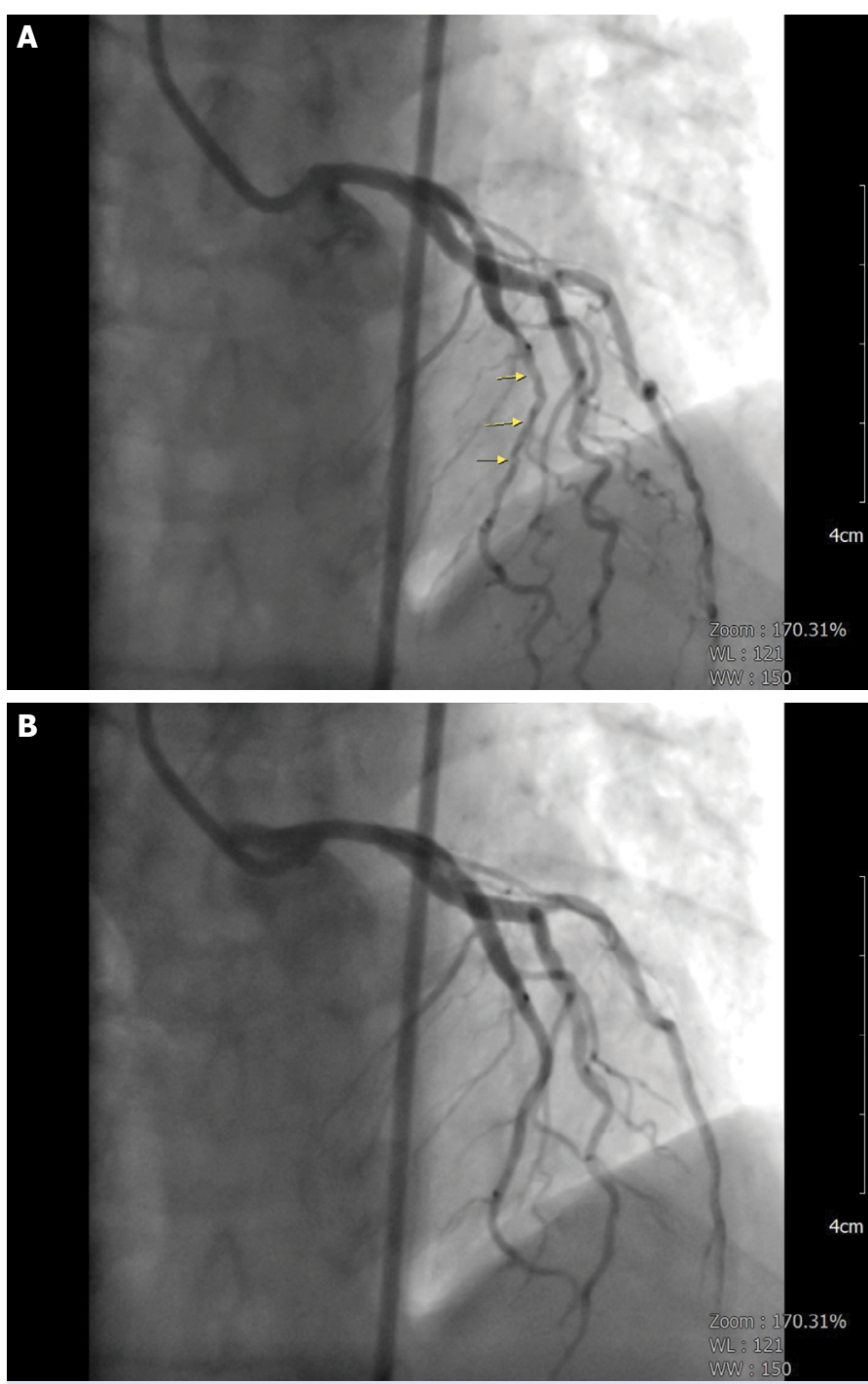

FIGURE 2. (A) Coronary angiography in the posteroanterior cranial projection during systole (arrows). (B) Coronary angiography in the posteroanterior cranial projection during diastole.

UIDEO 1. Coronary angiography in the posteroanterior cranial projection during diastole and systole. There is dynamic compression of the midportion of the left anterior descending artery during systole.

Informed Consent: Written informed consent was obtained from the patient for the publication of the case report and the accompanying images.

Conflict of Interest: No conflict of interest was declared by the authors.

Financial Disclosure: The authors declared that this study has received no financial support.

Authorship Contributions: Concept - SC, MOG; Design - SC; Supervision - SC, LO; Materials - SC, LO; Data collection and/or processing - SC, LO; Analysis and/or interpretation - SC, MOG; Literature review - SC, LO; Writing - SC, MOG; Critical review - MOG, LO. 


\section{REFERENCES}

1. Möhlenkamp S, Hort W, Ge J, Erbel R. Update on myocardial bridging. Circulation 2002;106:2616-22. [CrossRef]

2. Corban MT, Hung OY, Eshtehardi P, Rasoul-Arzrumly E, McDaniel M, Mekonnen G, et al. Myocardial bridging: contemporary understanding of pathophysiology with implications for diagnostic and therapeutic strategies. J Am Coll Cardiol 2014;63:2346-55. [CrossRef]

3. Eggebrecht H, Von Birgelen C, Ge J, Baumgart D, Erbel R. Postextrasystolic potentiation of vessel compression in myocardial bridging: detection by intravascular sonography. J Clin Ultrasound
2002;30:312-6. [CrossRef]

4. Berry JF, von Mering GO, Schmalfuss C, Hill JA, Kerensky RA. Systolic compression of the left anterior descending coronary artery: A case series, review of the literature, and therapeutic options including stenting. Cath Cardiovasc Intervent 2002;56:58-63. [CrossRef]

5. Tarantini G, Migliore F, Cademartiri F, Fraccaro C, Iliceto S. Left Anterior Descending Artery Myocardial Bridging: A Clinical Approach. J Am Coll Cardiol 2016;68:2887-99. [CrossRef]

6. Masuda T, Ishikawa Y, Akasaka Y, Itoh K, Kiguchi H, Ishii T. The effect of myocardial bridging of the coronary artery on vasoactive agents and atherosclerosis localization. J Pathol 2001;193:408-14. [CrossRef] 